Y. C. Minh and E. F. van Dishoeck, eds.

\title{
Crossed Molecular Beam Experiments of Radical-Neutral Reactions Relevant to the Formation of Hydrogen Deficient Molecules in Extraterrestrial Environments
}

\author{
R. I. Kaiser ${ }^{1}$, N. Balucani ${ }^{2}$, O. Asvany ${ }^{3}$, and Y. T. Lee \\ Institute of Atomic and Molecular Sciences, 1, Section 4, Roosevelt Rd., \\ 107 Taipei, Taiwan, ROC
}

\begin{abstract}
During the last 5 years, laboratory experiments relevant to the formation of carbon-bearing molecules in extraterrestrial environments have been performed employing the crossed molecular beam technique and a high intensity source of ground state atomic carbon, $\mathrm{C}\left({ }^{3} P_{j}\right)$. These investigations unraveled for the first time detailed information on the chemical reaction dynamics, involved collision complexes and intermediates, and - most important - reaction products of neutral-neutral reactions. Here, we extend these studies even further, and report on very recent crossed beam experiments of cyano radical, $\mathrm{CN}\left({ }^{2} \Sigma^{+}\right)$, reactions with unsaturated hydrocarbons to form nitriles in extraterrestrial environments and Saturn's moon Titan. Further, preliminary results on reactions of small carbon clusters $\mathrm{C}_{2}\left({ }^{1} \Sigma_{g}^{+}\right)$, and $\mathrm{C}_{3}\left({ }^{1} \Sigma_{g}^{+}\right)$with acetylene, ethylene, and methylacetylene to synthesize hydrogen-deficient carbonbearing molecules are presented.
\end{abstract}

\section{Introduction}

In the gas phase of the interstellar medium (ISM), about $97 \%$ of all molecular species are neutral, whereas only $3 \%$ are ions (Scheffler \& Elsässer 1988). These ions, radicals, and closed shell molecules are predominantly confined to distinct interstellar environments such as interstellar clouds, hot cores, and circumstellar envelopes of dying carbon and oxygen stars. Diffuse (hot) clouds have number densities $n$ of about 100 molecules $\mathrm{cm}^{-3}$ and average translational temperatures $T$ of $\approx 100 \mathrm{~K}$, whereas dense (cold, dark, molecular) clouds are characterized by $n=10^{3}-10^{6} \mathrm{~cm}^{-3}$ and $T=10-40 \mathrm{~K}$. Hot cores of molecular clouds have typical number densities up to $10^{7} \mathrm{~cm}^{-3}$ and temperatures reaching up to $300 \mathrm{~K}$. In the outer photosphere of carbon stars, temperatures can rise up to $4000 \mathrm{~K}$ (Alksne et al. 1991) and a more complex, even higher temperature chemistry is expected.

\footnotetext{
${ }^{1}$ Also: Department of Physics, Technical University Chemnitz, 09107 Chemnitz, Germany; and Department of Physics, National Taiwan University, Taipei, 106, ROC

${ }^{2}$ Visiting scientist. permanent address: Dipartimento di Chimica, Universitá di Perugia, 06123 Perugia, Italy

${ }^{3}$ Also: Department of Physics, Technical University Chemnitz, 09107 Chemnitz, Germany
} 
Up to now, about 114 molecular species ranging from the simplest diatomic molecule $\mathrm{H}_{2}$ to the most complex molecule $\mathrm{HC}_{11} \mathrm{~N}$ have been detected in these environments. However, despite large fractional abundances of, e.g. $\mathrm{C}_{3} \mathrm{H}$ and $\mathrm{C}_{3} \mathrm{H}_{2}$, up to $10^{-8}$ relative to atomic hydrogen, well-defined synthetic mechanisms to synthesize even these abundant species have not been fully resolved yet. Before we can elucidate their reaction pathways, we have to get familiar with the physical conditions under which the chemical reaction can occur. Here, the kinetic energy of interstellar species is confined to typically $0.8 \mathrm{~kJ} \mathrm{~mol}^{-1}$ (diffuse clouds) and $0.08 \mathrm{~kJ} \mathrm{~mol}^{-1}$ (dark, molecular clouds) on average, and hence gas-phase reactions under thermodynamical equilibrium conditions must be a) exothermic or only slightly endothermic, b) should exhibit little or no entrance barriers, and c) must involve only two body collisions. A three-body-reaction happens only once in a few $10^{9}$ years and can be neglected considering mean interstellar cloud lifetimes of $10^{6}$ years. Based on these restrictions, early chemical equilibrium models of interstellar chemistry focus solely on ion-molecule reactions, radiative associations, as well as dissociative recombination between cations and electrons to explain interstellar chemistry (Herbst \& Klemperer 1973). However, this approach fails to reproduce the formation of complex, carbon-bearing species and most of the fractional abundances of hydrocarbon isomers.

It is now well accepted that fast and barrier-less neutral - neutral reactions can form molecules detected in the interstellar medium, such as the well known linear and cyclic $\mathrm{C}_{3} \mathrm{H}$ isomers upon the reaction of atomic carbon, $\mathrm{C}\left({ }^{3} P_{j}\right)$, with acetylene, $\mathrm{C}_{2} \mathrm{H}_{2}$ (Kaiser et al. 1996a). Recently, various neutral - neutral reactions relevant to the formation of carbon containing molecules in the interstellar medium were performed elucidating the chemical dynamics and reaction products employing the crossed beam technique. These investigations provided collision energy dependent differential cross sections of a number of reactions of atomic carbon in its ${ }^{3} P_{j}$ electronic ground state with unsaturated hydrocarbons and $\mathrm{H}_{2} \mathrm{~S}$ (see Table 1 for summary).

\begin{tabular}{|c|c|c|c|c|}
\hline Reaction & Reactants & & Products & Ref. \\
\hline (1) & $\mathrm{C}\left({ }^{3} P_{j}\right)+\mathrm{C}_{2} \mathrm{H}_{2}\left({ }^{1} \Sigma_{g}^{+}\right)$ & $\rightarrow$ & $1-C_{3} H\left({ }^{2} \Pi_{1 / 2}\right) / c-C_{3} H\left({ }^{2} B_{2}\right)+H\left({ }^{2} S_{1 / 2}\right)$ & a \\
\hline (2) & $\mathrm{C}\left({ }^{3} P_{j}\right)+\mathrm{C}_{2} \mathrm{H}_{4}\left({ }^{1} \mathrm{~A}_{g}\right)$ & $\rightarrow$ & $\mathrm{C}_{3} \mathrm{H}_{3}\left({ }^{2} \mathrm{~B}_{2}\right)+\mathrm{H}\left({ }^{2} \mathrm{~S}_{1 / 2}\right)$ & b \\
\hline (3) & $\mathrm{C}\left({ }^{3} P_{j}\right)+\mathrm{CH}_{3} \mathrm{CCH}\left({ }^{1} \mathrm{~A}_{1}\right)$ & $\rightarrow$ & $\mathrm{n}-\mathrm{C}_{4} \mathrm{H}_{3}\left({ }^{2} \mathrm{~A}^{\prime}\right)+\mathrm{H}\left({ }^{2} \mathrm{~S}_{1 / 2}\right)$ & c \\
\hline (4) & $\mathrm{C}\left({ }^{3} P_{j}\right)+\mathrm{C}_{3} \mathrm{H}_{6}\left({ }^{1} \mathrm{~A}^{\prime}\right)$ & $\rightarrow$ & $\mathrm{C}_{4} \mathrm{H}_{5}\left({ }^{2} \mathrm{~B}_{2} /{ }^{2} \mathrm{~A}^{\prime \prime}\right)+\mathrm{H}\left({ }^{2} \mathrm{~S}_{1 / 2}\right)$ & d \\
\hline (5) & $\mathrm{C}\left({ }^{3} P_{j}\right)+\mathrm{H}_{2} \mathrm{CCCH}_{2}\left({ }^{1} \mathrm{~A}_{1}\right)$ & $\rightarrow$ & $\mathrm{n}-\mathrm{C}_{4} \mathrm{H}_{3}\left({ }^{2} \mathrm{~A}^{\prime}\right)+\mathrm{H}\left({ }^{2} \mathrm{~S}_{1 / 2}\right)$ & c \\
\hline (6) & $\mathrm{C}\left({ }^{3} P_{j}\right)+\mathrm{C}_{6} \mathrm{H}_{6}\left({ }^{1} \mathrm{~A}_{1} g\right)$ & $\rightarrow$ & $\mathrm{c}-\mathrm{C}_{7} \mathrm{H}_{5}\left({ }^{2} \mathrm{~B}_{2}\right)+\mathrm{H}\left({ }^{2} \mathrm{~S}_{1 / 2}\right)$ & e \\
\hline (7) & $\mathrm{C}\left({ }^{3} P_{j}\right)+\mathrm{CH}_{3} \mathrm{CCCH}_{3}\left({ }^{1} \mathrm{~A}_{1}\right)$ & $\rightarrow$ & $\mathrm{CH}_{3} \mathrm{CCCCH}_{2}\left({ }^{2} \mathrm{~A}^{\prime}\right)+\mathrm{H}\left({ }^{2} \mathrm{~S}_{1 / 2}\right)$ & \\
\hline (8) & $\mathrm{C}\left({ }^{3} P_{j}\right)+\mathrm{C}_{2} \mathrm{H}_{3}\left({ }^{2} \mathrm{~A}^{\prime}\right)$ & $\rightarrow$ & $\mathrm{C}_{3} \mathrm{H}_{2}+\mathrm{H}\left({ }^{2} \mathrm{~S}_{1 / 2}\right)$ & \\
\hline (9) & $\mathrm{C}\left({ }^{3} P_{j}\right)+\mathrm{C}_{3} \mathrm{H}_{3}\left({ }^{2} \mathrm{~B}_{2}\right)$ & $\rightarrow$ & $\mathrm{HCCCCH}\left({ }^{1} \Sigma_{g}^{+}\right)+\mathrm{H}\left({ }^{2} \mathrm{~S}_{1 / 2}\right)$ & $\mathrm{f}$ \\
\hline (10) & $\mathrm{C}\left({ }^{3} P_{j}\right)+\mathrm{H}_{2} \mathrm{~S}\left({ }^{1} \mathrm{~A}_{1}\right)$ & $\rightarrow$ & $\mathrm{HCS}\left({ }^{2} \mathrm{~A}^{\prime}\right)+\mathrm{H}\left({ }^{2} \mathrm{~S}_{1 / 2}\right)$ & $\mathrm{g}$ \\
\hline
\end{tabular}

References: a. Kaiser et al. (1996b); b. Kaiser et al. (1996a); c. Kaiser et al. (1996c); d. Kaiser et al. (1997a); e. Kaiser et al. (1999a); f. Kaiser et al. (1997b); g. Kaiser et al. (1999b)

The explicit identification of the carbon-hydrogen exchange under single collision conditions opens a versatile, one step pathway to free hydrocarbon radicals in extraterrestrial environments and even in our solar system. This mechanism can be employed further to predict large scale concentrations of hitherto unob- 
served interstellar radicals such as $\mathrm{C}_{3} \mathrm{H}_{3}, \mathrm{n}-\mathrm{C}_{4} \mathrm{H}_{3}$, and $\mathrm{C}_{4} \mathrm{H}_{5}$, once interstellar regions of atomic carbon overlapping with $\mathrm{C}_{2} \mathrm{H}_{4}, \mathrm{C}_{3} \mathrm{H}_{4}$, or $\mathrm{C}_{3} \mathrm{H}_{6}$ concentrations are charted. The present paper extends these crossed molecular beam investigations and compiles results on very recent reactions of cyano radicals in their vibrational ground state, $\mathrm{CN}\left({ }^{2} \Sigma^{+}\right)$, with unsaturated hydrocarbons, namely acetylene (reaction CN1), ethylene (reaction CN2), methylacetylene (reaction CN3), allene (reaction CN4), dimethylacetylene (reaction CN5), and benzene (reaction CN6) to form unsaturated nitriles in the interstellar medium. A second set of experiments reports on systematic studies on the formation of hydrogen-poor hydrocarbon molecules, i.e. reactions of carbon clusters $\mathrm{C}_{2}\left({ }^{1} \Sigma_{g}^{+}\right)$and $\mathrm{C}_{3}\left({ }^{1} \Sigma_{g}^{+}\right)$ with small unsaturated hydrocarbons, namely acetylene, ethylene, and methylacetylene. These experiments yield the first doubly differential reactive cross sections of carbon cluster reactions, supply valuable information on the involved potential energy surfaces to form hydrogen deficient carbon-containing species, supply a data base of product isomers for a refined modeling of interstellar chemistry, and yield generalized concepts based on the chemical dynamics to predict formation zones of hitherto unobserved interstellar molecules to guide the search of astronomers.

\section{Experimental and Data Analysis}

Our gas phase experiments were performed under single collision conditions employing the crossed molecular beam approach (Lee 1987). One of the basic ideas behind this experimental setup is that if, for example, a bimolecular reaction of two reactants $\mathrm{A}$ and $\mathrm{BC}$ takes place via a complex, $\mathrm{A}+\mathrm{BC} \rightarrow[\mathrm{ABC}]^{*} \rightarrow$ $\mathrm{AB}+\mathrm{C}$, one species $\mathrm{A}$ reacts only with one species $\mathrm{BC}$. A collisional stabilization or successive reaction of the initially formed $[A B C]^{*}$ intermediate or successive reaction of reaction products with a third molecule are excluded since the density of the reactants can be selected such that ternary encounters are absent. Therefore, the single collision condition represents an excellent simulation of the low density regions in the interstellar medium, even in hot cores and outflows of carbon stars. Second, highly unstable and reactive radicals can be prepared under well-defined conditions such as internal states, velocity spread, velocity, and hence collision energy, and reaction products with unknown spectroscopic properties can be probed. We like to stress that the experiments are performed at well-defined collision energies, and not at a fixed collision temperature, as is the case in chemical reactions in, for example, fast flow reactors or low temperature kinetic studies (Smith 1997). Third, we have to take into consideration that a variety of structural isomers can contribute to the reaction product. Here, the knowledge of intimate chemical dynamics of a reaction can be employed to elucidate the product isomer(s) and involved reaction intermediates - if any.

In detail, the $35^{\prime \prime}$ crossed molecular beam machine consists of two source chambers fixed at $90^{\circ}$ crossing angle, a stainless steel scattering chamber, and an ultra high vacuum-tight, rotatable triply differentially pumped quadrupole mass spectrometric detector (see Figure 1). The scattering chamber is evacuated by oil free turbo pumps to about $10^{-7}$ torr. In the primary source, a pulsed supersonic cyano $\mathrm{CN}\left(\mathrm{X}^{2} \Sigma^{+}\right)$radical beam, in which $\mathrm{CN}$ radicals are in the vibrational ground state, is generated in situ by laser ablation of graphite at 266 

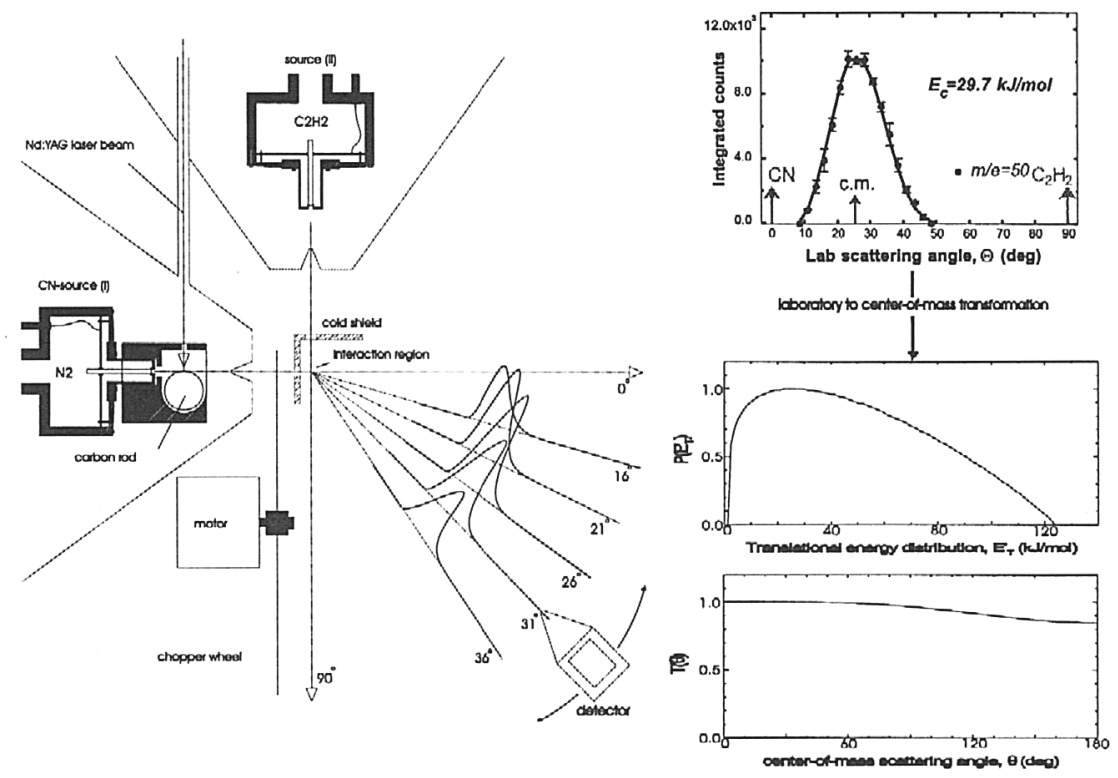

Figure 1. Schematic top view of the source chambers, chopper wheel, and interaction region together with the transformation from the laboratory $(\mathrm{LAB})$ to the center-of-mass $(\mathrm{CM})$ reference frame.

$\mathrm{nm}$ and subsequent seeding of the liberated species in molecular nitrogen which also serves as a reactant (Kaiser et al. 1999c). In a second set of experiments, $\mathrm{C}_{2}\left(\mathrm{X}^{1} \Sigma_{g}^{+}\right)$and $\mathrm{C}_{3}\left(\mathrm{X}^{1} \Sigma_{g}^{+}\right)$clusters were generated by laser ablation of graphite and seeding the ablated clusters in neat helium carrier gas (Kaiser \& Suits 1995). As a detector we use a triply differentially pumped detector consisting of a liquid nitrogen cooled electron impact ionizer followed by a quadrupole mass selector, and a Daly-type detector.

The reactively scattered species are analyzed at different laboratory angles $\Theta$ and at distinct mass to charge ratios $(\mathrm{m} / \mathrm{e})$ in the time-of-flight (TOF) mode, cf. Fig. 1. This means we record the intensity $I$ of an ion at $m / e$ versus the flight time $t$ choosing the pulse of a photodiode attached to the chopper wheel as a well-defined zero-time for the experiments to yield a TOF spectrum $I(\Theta, t)$. We can integrate TOF spectra of one mass-to-charge ratio at different laboratory angles and thus obtain the angular distribution of a product in the laboratory frame (LAB). However, for the physical interpretation of the reactive scattering data it is crucial to transform the laboratory data into the center-of-mass (CM) system. Here, we employ a forward-convolution routine to fit the TOF spectra $I(\Theta, t)$ at different laboratory angles and the LAB distribution. This procedure initially guesses the angular flux distribution $T(\theta)$ and the translational energy flux distribution $P\left(E_{T}\right)$ in the CM frame assuming mutual independence. Here, $\theta$ defines the scattering angle in the $\mathrm{CM}$ system measured from the primary beam and $E_{T}$ the CM translational energy. Then, TOF spectra and the LAB distribution are calculated from these $T(\theta)$ and $P\left(E_{T}\right)$ accounting for the velocity and angular spread of both beams, the detector acceptance angle, and 


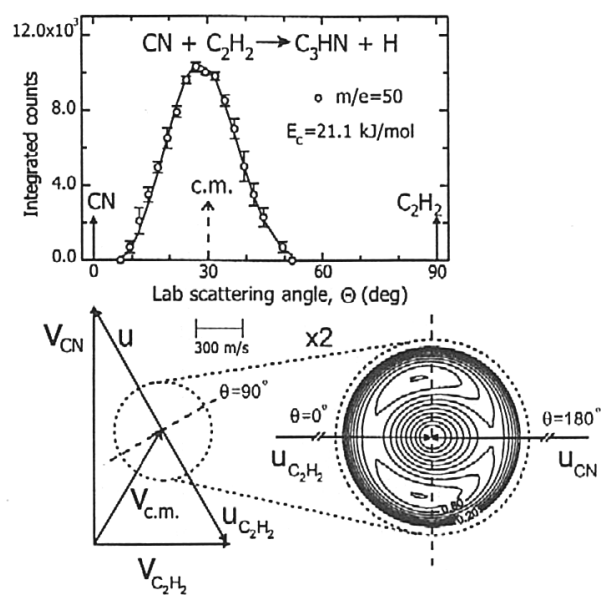

Figure 2. Laboratory angular distribution for the $\mathrm{CN}\left(\mathrm{X}^{2} \Sigma^{+}\right)+\mathrm{C}_{2} \mathrm{H}_{2}$ reaction of the $\mathrm{C}_{3} \mathrm{HN}$ product at a collision energy of $21.1 \mathrm{~kJ} \mathrm{~mol}^{-1}$ together with the most probable Newton diagram and flux contour map of the $\mathrm{C}_{3} \mathrm{HN}$ product.

the ionizer length. Both $T(\theta)$ and $P\left(E_{T}\right)$ are refined until a reasonable fit of the experimental data is achieved (see Kaiser et al. 1998 for details). The ultimate output of our experiments is the generation of a product flux contour map which reports the differential cross section (the intensity of the reactively scattered products), $I(\theta, u) \sim P(u) \times T(\theta)$, as a function of angle $\theta$ and product center-of-mass velocity $u$. The contour map serves as an image of the reaction and contains all the information of the scattering process.

\subsection{CN reactions}

In all investigated reactions $(\mathrm{CN} 1)$ - $(\mathrm{CN} 6)$, the $\mathrm{CN}$ versus $\mathrm{H}$ exchange channel was detected. We observed reactive scattering signals at mass-to-charge ratios $m / e=51\left(\mathrm{C}_{3} \mathrm{HN}\right.$, reaction $\left.\mathrm{CN} 1\right), m / e=53\left(\mathrm{C}_{3} \mathrm{H}_{3} \mathrm{~N}\right.$, reaction $\left.\mathrm{CN} 2\right), m / e=65$ $\left(\mathrm{C}_{4} \mathrm{H}_{3} \mathrm{~N}\right.$, reactions $\mathrm{CN} 3$ and $\left.\mathrm{CN} 4\right), m / e=79\left(\mathrm{C}_{5} \mathrm{H}_{5} \mathrm{~N}\right.$, reaction $\left.\mathrm{CN} 5\right)$, and $m / e=$ $103\left(\mathrm{C}_{7} \mathrm{H}_{5} \mathrm{~N}\right.$, reaction $\left.\mathrm{CN} 6\right)$. TOF flight spectra of lower $m / e$ ratios were recorded as well, but could be fit with the same center-of-mass functions as the parent ion. This finding indicates that those fragments originate in cracking of the parent molecule in the electron impact ionizer. Despite an intensive search, no radiative association channel was found experimentally. Figs. 2-4 show the experimental LAB distributions and the calculated curves using the center-of-mass best-fit functions. The lower parts of these figures depict the velocity vector diagram (Newton diagram) together with the contour maps of the heavy products. Selected TOF spectra of the products at the CM angles are shown in Fig. 5.

To get a picture of the energetics and hence the reaction products, we must take a closer look at the center-of-mass velocity $u$ of the products; here, all the information from $u$ translates to information on the translational energy and $P\left(E_{T}\right)$. To elucidate the chemical reaction dynamics, two properties of the $I(\theta, u)$ are worthwhile studying. First, if the energetics of the product isomers are well separated, the maximum translational energy $E_{\text {max }}$ can be used to identify the nature of the products. Here, $E_{\max }$ is simply the sum of the reac- 

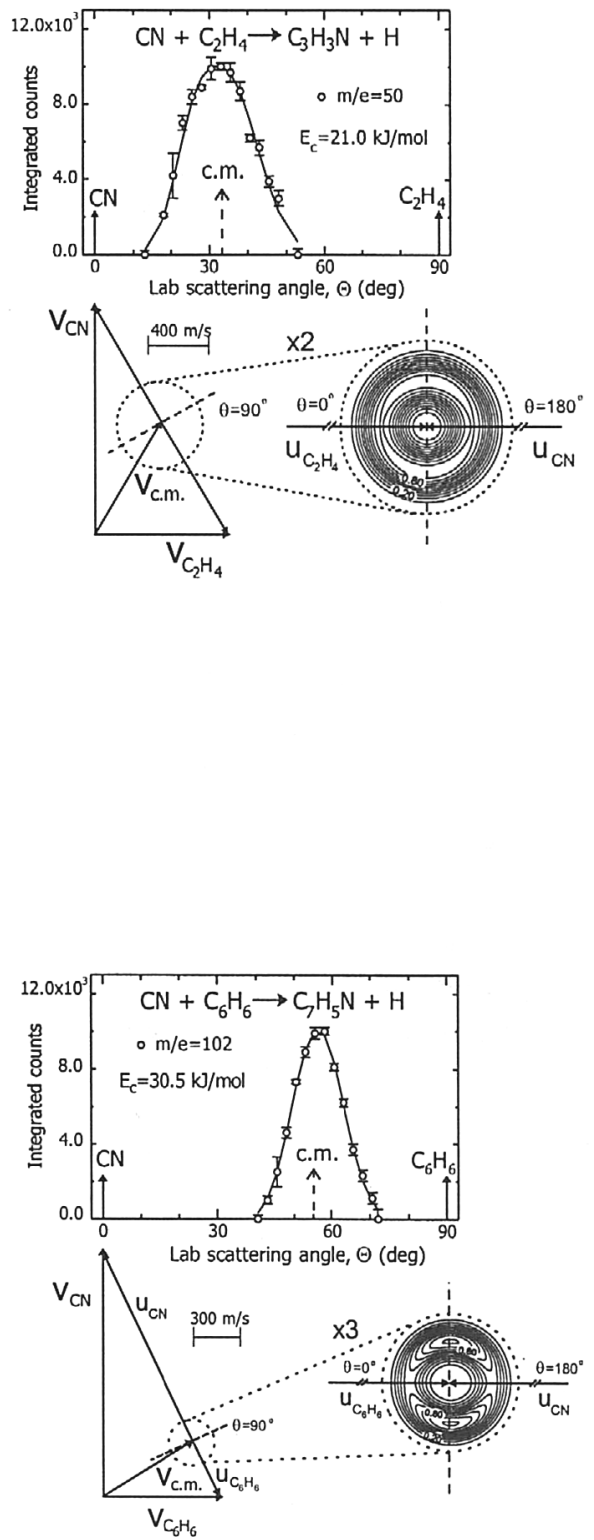

Figure 3. Laboratory angular distribution for the $\mathrm{CN}\left(\mathrm{X}^{2} \Sigma^{+}\right)+\mathrm{C}_{2} \mathrm{H}_{4}$ reaction of the $\mathrm{C}_{2} \mathrm{H}_{3} \mathrm{CN}$ product at a collision energy of 21.0 $\mathrm{kJ} \mathrm{mol}{ }^{-1}$ together with the most probable Newton diagram and flux contour map of the $\mathrm{C}_{2} \mathrm{H}_{3} \mathrm{CN}$ product.

Figure 4. Laboratory angular distribution for the $\mathrm{CN}\left(\mathrm{X}^{2} \Sigma^{+}\right)+\mathrm{C}_{6} \mathrm{H}_{6}$ reaction of the $\mathrm{C}_{6} \mathrm{H}_{5} \mathrm{CN}$ isomer at a collision energy of 30.5 $\mathrm{kJ} \mathrm{mol}{ }^{-1}$ together with the most probable Newton diagram and flux contour map of the $\mathrm{C}_{6} \mathrm{H}_{5} \mathrm{CN}$ product. 


\section{Time-Of-Flight Spectra}

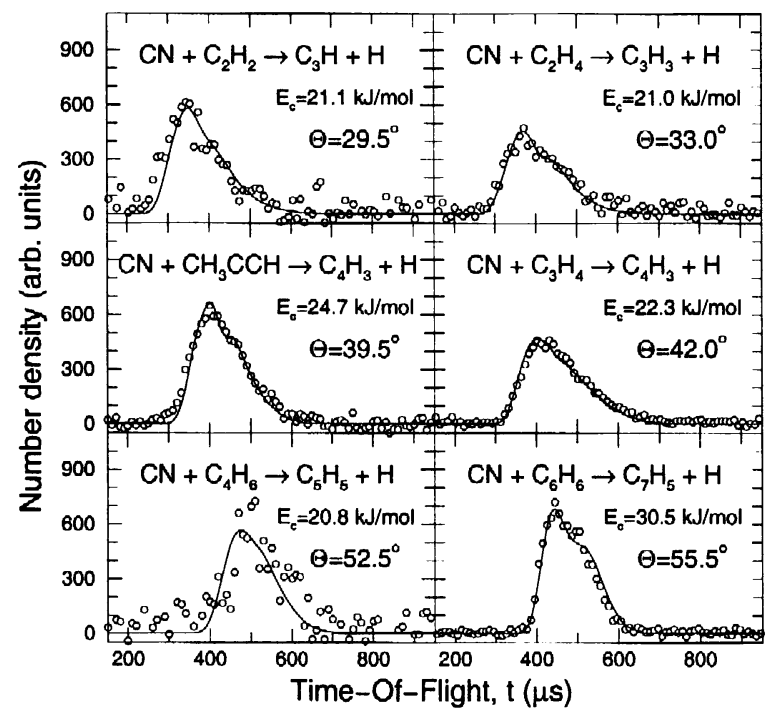

Figure 5. Selected time-of-flight data recorded at the center-of-mass angle of the crossed beam reactions $(\mathrm{CN} 1)-(\mathrm{CN} 6)$. The dots indicate the experimental data, the solid lines are the calculated fits.

tion exothermicity and the collision energy in our experiments. Therefore, if we subtract the collision energy from the experimentally determined $E_{\max }$ we simply get the exothermicity of the reaction. Hence we find that reactions (CN1) (CN6) must be exothermic by $80-110,90-100,65-110,85-95,70-110$, and $80-90 \mathrm{kJmol}^{-1}$. Based on the flux contour maps we find that at least the products cyanoacetylene (HCCCN, reaction $\mathrm{CN} 1)$, vinylcyanide $\left(\mathrm{C}_{2} \mathrm{H}_{3} \mathrm{CN}\right.$, reaction CN2), 1,1-cyanomethylallene $\left(\mathrm{CH}_{3} \mathrm{CNCCCH}_{2}\right.$, reaction $\left.\mathrm{CN} 5\right)$, and cyanobenzene $\left(\mathrm{C}_{6} \mathrm{H}_{5} \mathrm{CN}\right.$, reaction $\left.\mathrm{CN} 6\right)$ are formed. In case of reactions (CN3) and (CN4), the energetically accessible isomers cyanoallene $\left(\mathrm{CNHCCCH}_{2}\right)$ and cyanomethylacetylene $\left(\mathrm{CH}_{3} \mathrm{CCCN}\right)$ are separated by about $15-20 \mathrm{kJmol}^{-1}$, and cannot be discriminated yet. In case of reaction (CN3), we repeated the crossed beam experiments with the partially deuterated methylacetylene, $\mathrm{CD}_{3} \mathrm{CCH}$, and found a $\mathrm{H}$ atom as well as $\mathrm{D}$ atom loss channel. This indicates that both isomers $\mathrm{CH}_{3} \mathrm{CCCN}$ and $\mathrm{H}_{2} \mathrm{CCCHCN}$ are formed in reaction (CN3). Second, the fraction of energy released into translational degrees of freedom of $30-35 \%$ in our systems suggests that the chemical reaction dynamics are governed by an initial collision complex formation, i.e., a covalently bound intermediate from reaction of the unsaturated hydrocarbon with the $\mathrm{CN}$ radical. This has very important implications to planetary chemistry. In our crossed beam reactions, the life time of these intermediates is of the order of $10^{-12} \mathrm{~s}$; due to our single collision conditions, a third body collision cannot stabilize this internally excited intermediate. In denser planetary atmospheres, however, this three-body-reaction could take place to give a radical reaction product as well. 

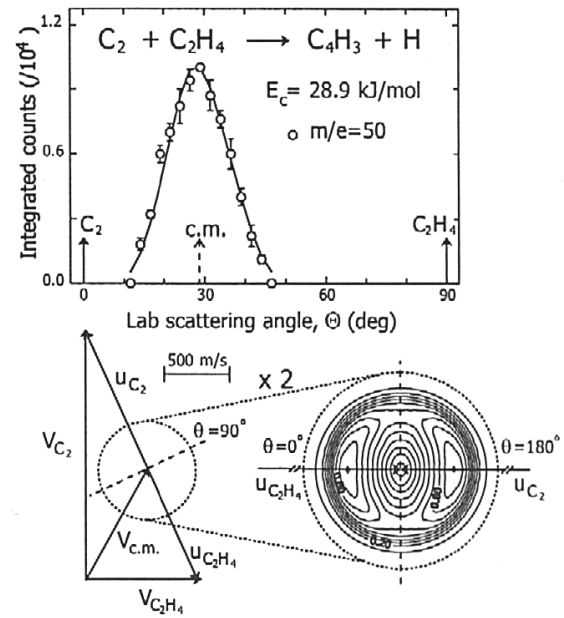

Figure 6. Laboratory angular distribution for the $\mathrm{C}_{2}+$ $\mathrm{C}_{2} \mathrm{H}_{4}$ reaction of the $\mathrm{C}_{4} \mathrm{H}_{3}$ product at a collision energy of $28.9 \mathrm{~kJ} \mathrm{~mol}^{-1}$ together with the most probable Newton diagram and flux contour map of the $\mathrm{C}_{4} \mathrm{H}_{3}$ product

\section{Results}

\subsection{CN reactions}

The angular distribution of the flux contour maps gives further information on the chemical reaction dynamics followed by reactions of the CN radicals with unsaturated hydrocarbons. These maps show the angular dependent flux distribution an observer would see if he moves with the center of mass system. Here, the direction of the $\mathrm{CN}$ beam is defined as $0^{\circ}$, and of that hydrocarbon beam as $180^{\circ}$, for a detailed discussion see Huang et al. (1999). Based on these considerations, it is obvious that all reactions $(\mathrm{CN} 1)$ - $(\mathrm{CN} 6)$ proceed via indirect scattering dynamics and involve a collision complex. We like to point out that in principle, all these collision complexes can be isolated in denser media such as the atmosphere of Titan and contribute directly to the chemistry in the troposphere, but not in the interstellar medium since the number density of both molecules is insufficient to give a three-body-reaction.

\section{2. $\mathbf{C}_{2} / \mathbf{C}_{3}$ reactions}

The data analysis of these reactions is still ongoing, and we like to report only preliminary results. Figure 6 shows the laboratory angular distribution and best fit together with the Newton diagram and flux contour map for the reaction of $\mathrm{C}_{2}$ with ethylene; representative TOF spectra are depicted at selected angles in Figure 7.

The reactions of $\mathrm{C}_{2}$ and $\mathrm{C}_{3}$ with unsaturated hydrocarbons acetylene, ethylene, and methylacetylene show striking differences. Crossed beam experiments of $\mathrm{C}_{2}$ depict the $\mathrm{C}_{2}$ versus $\mathrm{H}$ atom exchange pathway at collision energies even as low as $8 \mathrm{~kJ} \mathrm{~mol}^{-1}$ up to $39 \mathrm{~kJ} \mathrm{~mol}^{-1}$ to form $\mathrm{C}_{4} \mathrm{H}(m / e=49), \mathrm{C}_{4} \mathrm{H}_{3}(m / e=51)$, and $\mathrm{C}_{5} \mathrm{H}_{3}(m / e=63)$. Detailed studies of the reaction of $\mathrm{C}_{2}$ with partially deuterated $\mathrm{CD}_{3} \mathrm{CCH}$ showed that only the $\mathrm{D}$ atom is released; the $\mathrm{C}_{2} \mathrm{H}$ group is conserved throughout the reaction. In strong contrast to the $\mathrm{C}_{2}$ system, our experiments of $\mathrm{C}_{3}$ with acetylene, ethylene, and methylacetylene show reactive scattering signal for the $\mathrm{C}_{3}$ versus $\mathrm{H}$ atom exchange yielding $\mathrm{C}_{5} \mathrm{H}(m / e=61)$, 


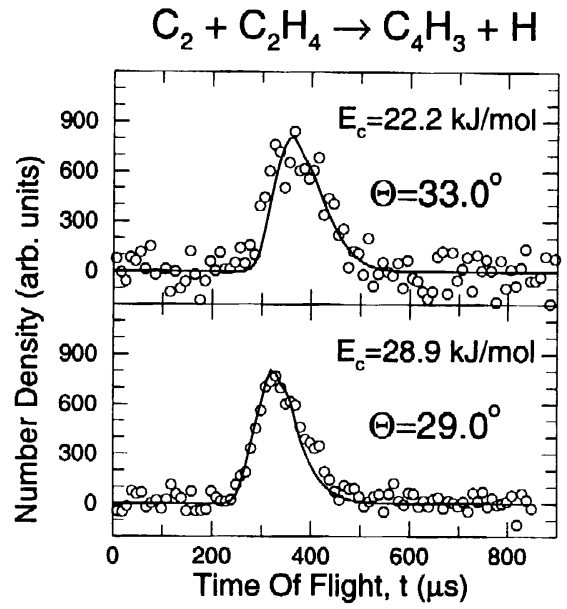

Figure 7. Selected time-offlight spectra of the $\mathrm{C}_{2}+$ $\mathrm{C}_{2} \mathrm{H}_{4}$ reaction as depicted in Figure 6.

$\mathrm{C}_{5} \mathrm{H}_{3}(m / e=63)$, and $\mathrm{C}_{6} \mathrm{H}_{3}(m / e=75)$, only if the collision energy is higher than $80 \pm 9 \mathrm{~kJ} \mathrm{~mol}^{-1}\left(\mathrm{C}_{3} / \mathrm{C}_{2} \mathrm{H}_{2}\right)$ and $56 \pm 4 \mathrm{~kJ} \mathrm{~mol}^{-1}\left(\mathrm{C}_{3} / \mathrm{C}_{2} \mathrm{H}_{4}\right)$. The threshold of the $\mathrm{C}_{3} / \mathrm{CH}_{3} \mathrm{CCH}$ system is currently under investigation and ranges between 49 and $98 \mathrm{~kJ} \mathrm{~mol}^{-1}$. In strong coincidence with the $\mathrm{C}_{2} / \mathrm{CH}_{3} \mathrm{CCH}$ system, the $\mathrm{H}$ atom loss appears only from the methyl group.

Detailed information on the reaction products can be given so far only on the $\mathrm{C}_{2} / \mathrm{C}_{2} \mathrm{H}_{4}$ reaction. Supported by RRKM and ab initio calculations, our experimental data are consistent with the formation of the $\mathrm{n}_{-} \mathrm{C}_{4} \mathrm{H}_{3}$ isomer. Further, at least the linear $\mathrm{C}_{4} \mathrm{H}$ and linear $\mathrm{C}_{5} \mathrm{H}$ isomers are formed in the $\mathrm{C}_{2} / \mathrm{C}_{2} \mathrm{H}_{2}$ and $\mathrm{C}_{3} / \mathrm{C}_{2} \mathrm{H}_{2}$ systems, respectively. The assignment of the other isomers and the interpretation of the $\mathrm{C}_{3} / \mathrm{C}_{3} \mathrm{H}_{4}$ as well as $\mathrm{C}_{2} / \mathrm{C}_{3} \mathrm{H}_{4}$ systems is still in progress.

\section{Reaction Pathways}

\subsection{CN reactions}

The reaction pathways leading to the unsaturated nitriles as detected in our experiments can be compiled as follows. Those readers who are interested in the detailed chemical dynamics are referred to Huang et al. (1999). The chemical dynamics of reactions $(\mathrm{CN} 1)$ - $(\mathrm{CN} 6)$ to form unsaturated nitriles are very similar. All reactions proceed via a complex and are initiated by an attack of the CN radical with the carbon atom to the $\pi$ electron density of the double or triple bond of the unsaturated hydrocarbon molecule, cf. Fig. 8. This process forms a new $\mathrm{C}-\mathrm{C} \sigma$ bond at the expense of a $\mathrm{C}-\mathrm{C} \pi$ bond leading to cis/trans $\mathrm{C}_{3} \mathrm{NH}_{2}$ radicals (reaction CN1), cyanoethyl radical (reaction CN2), cis and trans $\mathrm{CH}_{3} \mathrm{CC}(\mathrm{CN}) \mathrm{H}$ (reaction $\mathrm{CN} 3$ ), $\mathrm{H}_{2} \mathrm{CCCH}_{2}(\mathrm{CN})$ (reaction $\mathrm{CN} 4$ ), cis/trans $\mathrm{CH}_{3} \mathrm{CC}(\mathrm{CN}) \mathrm{CH}_{3}$ (reaction CN5), and $\mathrm{C}_{6} \mathrm{H}_{6}$ - $\mathrm{CN}$ (reaction CN6). As supported by electronic structure calculations, these reactions have no entrance barrier; further, all intermediates are bound in a potential energy well in the order of $300 \mathrm{kJmol}^{-1}$ with respect to the separated reactants. We like to stress that since methylacetylene is not symmetric, CN can attack either the $\alpha$ carbon 

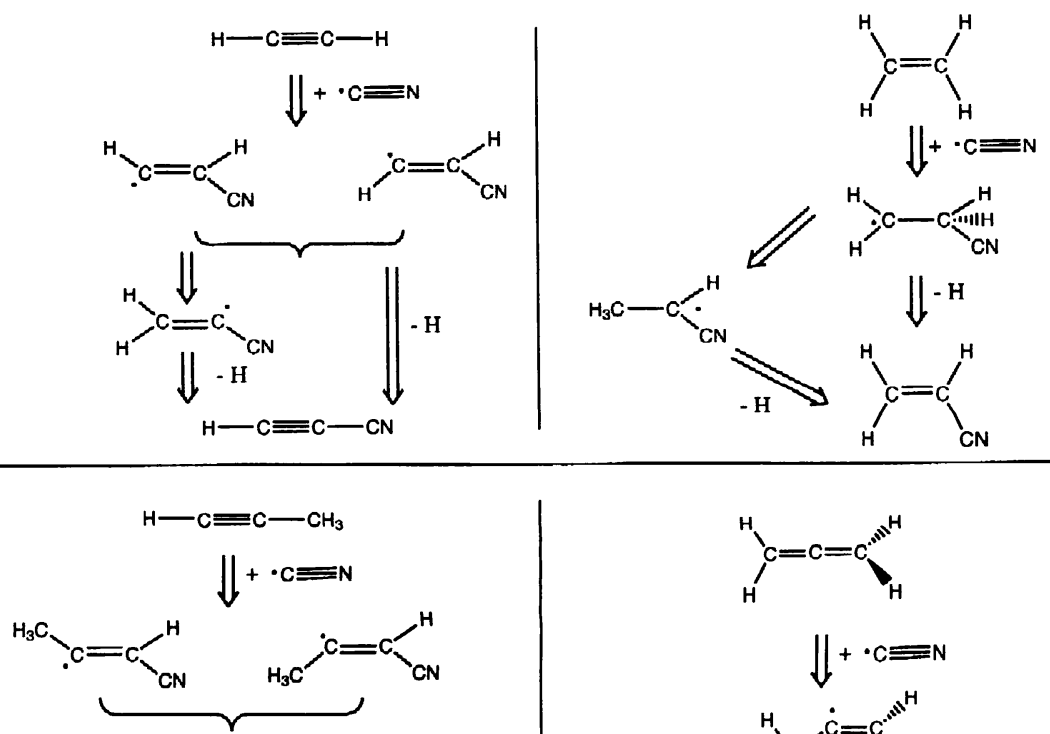

$-\mathrm{H} \downarrow$

$\downarrow-H$<smiles>[C+]C=C=C</smiles>

$\mathrm{H}_{3} \mathrm{C}-\mathrm{C}=\mathrm{C}-\mathrm{CN}$<smiles>C=C=C</smiles><smiles>C=C[C+]C(C=C)=CC#N</smiles><smiles>C=C=C[CH2+]</smiles><smiles>CC#C[C+]#N</smiles><smiles>CC=C(C)C#N</smiles>

$-\mathrm{H} \downarrow$<smiles>C=C=C[C+](C)C</smiles>

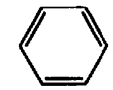

$\Downarrow+\cdot \mathrm{C} \equiv \mathrm{N}$
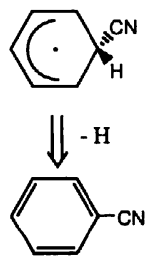

Figure 8. Schematic representation of the reaction $\mathrm{CN}\left(\mathrm{X}^{2} \Sigma^{+}\right)+$ $\mathrm{C}_{2} \mathrm{H}_{2}$ (top left), $\mathrm{C}_{2} \mathrm{H}_{4}$ (top right), $\mathrm{CH}_{3} \mathrm{CCH}$ (middle left), $\mathrm{H}_{2} \mathrm{CCCH}_{2}$ (middle right), $\mathrm{CH}_{3} \mathrm{CCCH}_{3}$ (bottom left), and $\mathrm{C}_{6} \mathrm{H}_{6}$ (bottom right). See text for details. 


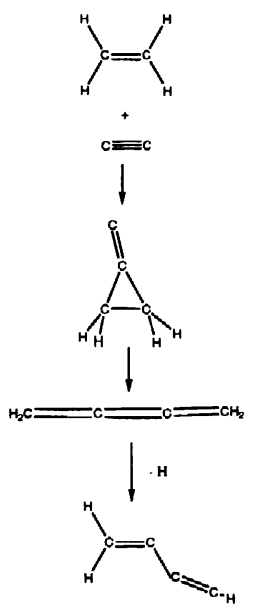

Figure 9. Schematic representation of the reaction $\mathrm{C}_{2}\left({ }^{1} \Sigma_{g}^{+}\right)+\mathrm{C}_{2} \mathrm{H}_{4}$. See text for details.

atom (the carbon atom to which the $\mathrm{CH}_{3}$ group is attached) or the $\beta$ position. Since the bulky methyl group reduces the cone of acceptance to the $\alpha$ position, $\mathrm{CN}$ should attack preferentially the $\beta$ carbon atom. Further, the $\beta$ position has a spin density higher than the $\alpha$ atom. This directs the attack of the $\mathrm{CN}$ radical to the $\beta$ position even further to form the cis and trans $\mathrm{CH}_{3} \mathrm{CC}(\mathrm{CN}) \mathrm{H}$ intermediate. As inferred from the angular part of the flux contour maps, the initially formed collision complexes have lifetimes of the order of at least $0.5 \mathrm{ps}$; all intermediates can undergo a carbon-hydrogen bond rupture to form cyanoacetylene ( $\mathrm{HCCCN}$, reaction $\mathrm{CN} 1)$, vinylcyanide $\left(\mathrm{C}_{2} \mathrm{H}_{3} \mathrm{CN}\right.$, reaction $\left.\mathrm{CN} 2\right)$, cyanoallene $\left(\mathrm{H}_{2} \mathrm{CCC}(\mathrm{CN}) \mathrm{H}\right)$ and cyanomethylacetylene $\left(\mathrm{CH}_{3} \mathrm{CCCN}\right.$; both reaction $\mathrm{CN} 3)$, cyanoallene $\left(\mathrm{HCCCH}_{2} \mathrm{CN}\right.$, reaction $\left.\mathrm{CN} 4\right)$, 1,1-cyanomethylallene $\left(\mathrm{CH}_{3} \mathrm{CCCNCH}_{2}\right.$, reaction $\left.\mathrm{CN} 5\right)$, and cyanobenzene $\left(\mathrm{C}_{6} \mathrm{H}_{5} \mathrm{CN}\right.$, reaction $\left.\mathrm{CN} 6\right)$. We like to stress that in reaction $\mathrm{CN} 1$ and $\mathrm{CN} 2$, a hydrogen shift prior to bond rupture is involved to a minor amount of $20 \%$.

\section{2. $\mathrm{C}_{2} / \mathrm{C}_{3}$ reactions}

Until now, only the detailed chemical dynamics of the $\mathrm{C}_{2} / \mathrm{C}_{2} \mathrm{H}_{4}$ reaction have been investigated. Our data combined with ab initio and statistical calculations indicate that $\mathrm{C}_{2}\left({ }^{1} \Sigma_{g}^{+}\right)$attacks the olefinic bond to form a three-membered intermediate which undergoes ring opening to butatriene followed by an $\mathrm{H}$ atom loss to form the $n-\mathrm{C}_{4} \mathrm{H}_{3}$ isomer, cf. Fig. 9 (Balucani et al. 1999). There is neither an entrance nor an exit barrier; the reaction exothermicity is calculated to 156 $\mathrm{kJmol}^{-1}$.

\section{Astrophysical Implications and Conclusions}

The crossed molecular beam experiments have been established as an elegant method to unravel the chemical reaction dynamics, involved complexes, and product isomers of radical - neutral reactions of the cyano radical, $\mathrm{CN}\left(\mathrm{X}^{2} \Sigma^{+}\right)$, with unsaturated hydrocarbon molecules $\mathrm{C}_{2} \mathrm{H}_{2}, \mathrm{C}_{2} \mathrm{H}_{4}, \mathrm{CH}_{3} \mathrm{CCH}, \mathrm{H}_{2} \mathrm{CCCH}_{2}$, 
$\mathrm{CH}_{3} \mathrm{CCCH}_{3}$, and $\mathrm{C}_{6} \mathrm{H}_{6}$ to form unsaturated nitriles. This is the first direct experimental proof that radical - neutral reactions of CN radicals with unsaturated hydrocarbons can form nitriles in the interstellar medium as well: here, all reactions have no entrance barrier, show an exit barrier well below the energy of the reactant molecules, and are exothermic. Therefore, these nitriles can be formed even in the coldest environments such as cold molecular clouds holding average translational temperatures of $10 \mathrm{~K}$. The $\mathrm{CN}$ versus $\mathrm{H}$ atom exchange channel under well-defined single collision conditions makes it even possible to predict the formation of hitherto unobserved nitriles: up to now, only $\mathrm{HC}$ $\mathrm{CCN}, \mathrm{C}_{2} \mathrm{H}_{3} \mathrm{CN}$, and $\mathrm{CH}_{3} \mathrm{CCCN}$ have been detected astronomically, but neither cyanoallene, $\mathrm{H}_{2} \mathrm{CCCCNH}$, nor cyanobenzene, $\mathrm{C}_{6} \mathrm{H}_{5} \mathrm{CN}$, were found. The latter two molecules might resemble ideal tracers of interstellar allene, $\mathrm{H}_{2} \mathrm{CCCH}_{2}$, and benzene, $\mathrm{C}_{6} \mathrm{H}_{6}$, since they are not accessible to radioastronomical detection because they lack a permanent dipole moment.

In addition, our preliminary data suggest that reactions of $\mathrm{C}_{2}\left({ }^{1} \Sigma_{g}^{+}\right)$with unsaturated hydrocarbons acetylene, ethylene, and methylacetylene have most likely no entrance barrier and are exothermic; therefore, they may occur even in dark molecular clouds. The reaction of $\mathrm{C}_{2}$ with $\mathrm{C}_{2} \mathrm{H}_{4}$ provides a barrierless, third synthetic route to hitherto unobserved interstellar $n-C_{4} \mathrm{H}_{3}$ isomers; previous studies of atomic carbon with allene and acetylene showed that the $\mathrm{n}-\mathrm{C}_{4} \mathrm{H}_{3}$ isomer is formed in these reactions as well. Thercfore, it is expected to be present in the interstellar medium. Its ground state is a bent structure, whereas the linear butatrienyl structure represents a transition state between two bent states, located only $255 \mathrm{~cm}^{-1}$ above the $\mathrm{n}-\mathrm{C}_{4} \mathrm{H}_{3}$. Since the coldest molecular clouds have average translational temperatures of about $10 \mathrm{~K}$ - about $7 \mathrm{~cm}^{-1}-\mathrm{n}-\mathrm{C}_{4} \mathrm{H}_{3}$ must be bent. The transition state is energetically not accessible. In hotter interstellar environments such as hot molecular cores with average translational temperatures of $200-300 \mathrm{~K}$ - about $140-210 \mathrm{~cm}^{-1}-\mathrm{C}_{4} \mathrm{H}_{3}$ radicals populating translational energies in the long tail of the Maxwell-Boltzmann distribution could overcome the barrier and these radical should be quasi linear. Radicals with lower kinetic energies must be bent. Therefore, the microwave spectrum of the $n-\mathrm{C}_{4} \mathrm{H}_{3}$ radical depends strongly on the temperature of the interstellar environments. Vice versa, recording these microwave spectra could serve as a probe to sample the temperature in these environments. Finally, we like to stress that especially the encounter of $\mathrm{C}_{2}$ with $\mathrm{C}_{2} \mathrm{H}_{2}$ is very interesting as it provides a single collision process in which two carbon atoms can be incorporated into a molecule to form an astronomically observed linear $\mathrm{C}_{4} \mathrm{H}$ isomer. To the best of our knowledge, no ion-molecule reaction can explain satisfactorily the formation of this isomer, and our results may be a compelling evidence that neutral-neutral reactions might synthesize $1-\mathrm{C}_{4} \mathrm{H}$. Further reactions such as that of $\mathrm{C}_{2}$ with diacetylene, $\mathrm{HCCCCH}$, are scheduled; the latter might form observed linear $\mathrm{C}_{6} \mathrm{H}$.

In strong contrast to the $\mathrm{CN}$ and $\mathrm{C}_{2}$ systems, all bimolecular reactions of $\mathrm{C}_{3}\left({ }^{1} \Sigma_{g}^{+}\right)$with acetylene, ethylene, and methylacetylene are endothermic and hence cannot proceed in cold molecular clouds. However, these processes are feasible at elevated temperatures such as in the outflow of carbon rich stars close to the photosphere. Here, the reaction of $\mathrm{C}_{3}$ with $\mathrm{C}_{2} \mathrm{H}_{2}$ is an excellent candidate to form the already observed $1-\mathrm{C}_{5} \mathrm{H}$ isomer. Our preliminary data on the $\mathrm{C}_{2} / \mathrm{C}_{3}$ reactions with acetylene suggest that the alternating $\mathrm{C}_{n} \mathrm{H}$ and $\mathrm{C}_{m} \mathrm{H}$ 
( $\mathrm{n}=$ even, $\mathrm{m}=$ odd $)$ abundances in distinct interstellar environments might be a result of distinct chemical reactivity and exothermicity; clearly, more experimental data especially on reactions of small carbon clusters with diacetylene and larger clusters with acetylene and diacetylene are crucial to get a more detailed, systematic correlation. These studies are in progress.

Acknowledgments. RIK is indebted the Deutsche Forschungsgemeinschaft (DFG) for a Habilitation fellowship (IIC1-Ka1081/3-1) and Prof. D. Gerlich (Technical University Chemnitz, Germany) for support. The work was supported by Academia Sinica and the Taiwanese Petrolcum Corporation.

\section{References}

Alksne, Z.K., Alksnis, A.K., \& Dzervitis, U.K. 1991, Properties of Galactic Carbon Stars (Orbit Book: Malabar)

Balucani, N., Mebel, A., Asvany, O., Chang, A.H.H., Lin, S.H., Lee, Y.T., \& Kaiser, R.I. 1999, J. Chem. Phys., submitted

Herbst, E. \& Klemperer, W. 1973, ApJ, 185, 505

Huang, L.C.L., Lee, Y.T., \& Kaiser, R.I. 1999, J. Chem. Phys., 110, 7119

Kaiser, R.I., Hahndorf, I., Huang, L.C.L., Lee, Y.T., Bettinger, H.F., Schleyer, P., \& Schaefer III, H.F. 1999a, J. Chem. Phys., 110, 6091

Kaiser, R.I., Lee, Y.T., \& Suits, A.G. 1996a, J. Chem. Phys., 105, 8705

Kaiser, R.I., Ochsenfeld, C., Head-Gordon, M., \& Lee Y.T. 1999b, J. Chem. Phys., 110, 2391

Kaiser, R.I., Ochsenfeld, C., Head-Gordon, M., Lee, Y.T., \& Suits, A.G. 1996b, Science, 274, 1508

Kaiser, R.I., Ochsenfeld, C., Stranges, D., Head-Gordon, M., \& Lee, Y.T. 1998, J. Chem. Soc. Farad. Discussion, 109, 183

Kaiser, R.I., Stranges, D., Bevsek, H.M., Lee, Y.T., \& Suits, A.G. 1997a, J. Chem. Phys., 106, 4945

Kaiser, R.I., Stranges, D., Lee, Y.T., \& Suits, A.G. 1996c, J. Chem. Phys., 105, 8721

Kaiser, R.I. \& Suits, A.G. 1995, Rev. Sci. Instr., 66, 5405

Kaiser, R.I., Sun, W., Suits, A.G., \& Lee, Y.T. 1997b, J. Chem. Phys., 107, 8713

Kaiser, R.I., et al. 1999c, Rev. Sci. Instr., 70, 4185

Lee, Y.T. 1987, Science, 236, 793

Scheffler, H. \& Elsässer, H. 1988, Physics of the Galaxy and Interstellar Matter (Springer: Berlin)

Smith, I.W.M. 1997, in Molecules in Astrophysics: Probes and Processes, ed. E.F. van Dishoeck (Kluwer: Dordrecht), 253

\section{Discussion}

E. Herbst: Could you possibly modify your apparatus to achieve lower collision energies?

R. I. Kaiser: If you provide us with enough money we certainly can do it! More seriously: our setup has two sources "fixed" at 90 degrees. The lowest 
collision energy we can get is about $4 \mathrm{~kJ} \mathrm{~mol}^{-1}$. For lower energies you need a machine in which the crossing angle and hence the collision energy can be reduced. There are three of these machines, i.e. K. Liu (Institute of atomic and molecular sciences, Taiwan), M. Costes (France), and Sims/Smith (England). However, these machines have no universal detector but spectroscopic, laserbased detection schemes.

T. Geballe: Your experiments simulate astrophysical conditions (in cold clouds) in the excitation temperatures of the molecules, but not in the kinetic temperatures of the molecules and the collision energies. Please comment on the astrophysical relevance.

R. I. Kaiser: The crossed beam experiments combined with state-of-the art ab initio calculations show unambiguously that the reactions of $\mathrm{CN}$ and $\mathrm{C}_{2} \mathrm{H}$ with unsaturated hydrocarbons, as well as $\mathrm{C}_{2}$ with $\mathrm{C}_{2} \mathrm{H}_{4}$ have no entrance barrier and are all exothermic. Further, the exit barrier is below the available energy. These criteria indicate that all reactions are feasible in cold clouds as well as in high temperature, inner circumstellar envelopes of carbon stars. Although our collision energies are higher than, e.g. in cold molecular clouds, the explicit identification of one isomer in the reactions with $\mathrm{C}_{2} \mathrm{H}_{2}, \mathrm{C}_{2} \mathrm{H}_{4}$, and $\mathrm{C}_{6} \mathrm{H}_{6}$ makes this crossed beams approach a universal experimental technique. Our results indicate that the formation of this one isomer is invariant on the collision energy! In the case of the reaction of $\mathrm{CN}$ and $\mathrm{C}_{2} \mathrm{H}$ with $\mathrm{CH}_{3} \mathrm{CCH}$ the situation is more complex, since we form two isomers. The branching ratios to these two isomers might depend on the collision energy, this is truc. Our experiments can predict which isomers can be formed, but it is not always possible to predict the energy dependent branching ratios. For these cases we are currently performing variational RRKM calculations to get the branching ratios in the temperature range from $10 \mathrm{~K}$ to $3000 \mathrm{~K}$. Second, our experiments show that all reactions of $\mathrm{C}_{3}$ with unsaturated hydrocarbons are endothermic and therefore cannot happen in cold clouds.

B. R. Rowe: How do you know precisely the internal states of your reactants (vibration, rotation, spin-orbit)? What is your energy resolution?

R. I. Kaiser: (1) Our energy resolution is in the most favorable case $3-5 \%$. (2) We have no means to investigate the spin orbit and rotational distribution right now. Since we have a supersonic expansion however and since all our experiments could be fitted with no excess energy, the molecules/radicals should be rotationally cold $(10-30 \mathrm{~K})$. Regarding the vibrational state distribution of e.g. the $\mathrm{CN}$ radical, we can select different parts of the pulsed beam. If one selects a fast part, one finds vibrationally-excited CN up to $v=5$. This can be correlated to an excess energy in our translational energy distributions in the center of mass frame. If one goes below $v=1900 \mathrm{~m} \mathrm{~s}^{-1}$ of the CN beam, all CN is in $v=0$ (see Kaiser et al. 1999c, in press). Further, the electronically excited states are too short-lived to be relevant in our exeriments with CN radicals. 